\title{
A novel technique for the surgical management of intraocular lens-capsular bag-capsular tension ring complex dislocation
}

\author{
LIN GUO, CONGYI WANG and XINGUANG YANG
}

\begin{abstract}
Department of Ophthalmology, Shaanxi Ophthalmic Medical Center, Xi'an No. 4 Hospital, Affiliated GuangRen Hospital, School of Medicine, Xi'an Jiao Tong University, Xi'an, Shaanxi 710004, P.R. China
\end{abstract}

Received July 4, 2016; Accepted November 4, 2018

DOI: $10.3892 /$ etm.2019.7224

\begin{abstract}
The present study describes a surgical technique for the management of complex dislocation and subluxation of the intraocular lens (IOL)-capsular bag (CB)-capsular tension ring (CTR) complex. During the procedure, one double-curved suture was inserted through the sclera into the posterior chamber, passing through the anterior capsules and then into the anterior chamber. The suture lines were pulled out from above and below the IOL-CB-CTR complex and a knot was tied $1 \mathrm{~mm}$ away from the corneoscleral incision. The IOL-CB-CTR complex was repositioned by adjusting the suture length. This novel method does not require explanting of the original IOL, allowing the IOL-CB-CTR complex to be immobilized in a simple and effective way.
\end{abstract}

\section{Introduction}

Dislocation or subluxation of the intraocular lens (IOL)-capsular bag (CB)-capsular tension ring (CTR) complex is a serious complication of cataract surgery (1). Spontaneous late in-the-bag IOL dislocation is commonly caused by trauma or pseudoexfoliation (1-5). Although the implantation of a CTR relieves zonular tension, the IOL-CB-CTR complex is also susceptible to dislocation and subluxation. The rate of surgical correction for dislocation or subluxation of the IOL-CB-CTR complex is $0.76 \%$ with an interval of $92.7 \pm 23.4$ months between the initial and reparative surgery (6).

Traditionally, dislocation of the IOL-CB-CTR complex has been treated by explanting the IOL-CTR through an incision in the scleral tunnel or corneal limbus, followed by implantation of a new IOL (7). However, several inherent problems are

Correspondence to: Professor Xinguang Yang, Department of Ophthalmology, Shaanxi Ophthalmic Medical Center, Xi'an No. 4 Hospital, Affiliated GuangRen Hospital, School of Medicine, Xi'an Jiao Tong University, 21 Jie Fang Road, Xi'an, Shaanxi 710004, P.R. China

E-mail: g-lin@126.com

Key words: intraocular lens-capsular bag-capsular tension ring complex, dislocation, subluxation, suturing associated with IOL explantation and replacement. The lens extraction incision must be $3-5 \mathrm{~mm}$ in length, and the IOL and CTR must be removed independently of each other. During the procedure, if either the IOL or CTR is dislocated posteriorly into the vitreous cavity, a vitrectomy may be necessary and a new IOL may be required be implanted at low intraocular pressure (8). Furthermore, IOL extraction and replacement is technically challenging and time-consuming. Complications accompanying this procedure include massive retinal hemorrhage, retinal detachment and intraocular infection.

The ideal surgical approach to correct dislocation or subluxation of the IOL-CB-CTR complex avoids any requirement for extraction of the IOL-CB-CTR complex, by resolving the subluxation with a small corneal incision (1).

The method proposed by the present study is quick, simple and minimally invasive. It involves the repositioning and fixation of the IOL through sutures. This may reduce intraocular pressure and prevent post-operative complications. The present study describes a simple and efficient technique for suturing the dislocated IOL-CB-CTR complex on the sclera, using 1-mm corneoscleral limbus incisions.

\section{Case study and surgical technique}

The current case study is a representative case. Five years and 10 months prior to presentation, a 59-year-old man with a history of chronic obstructive pulmonary disease was treated with phacoemulsification of the right eye, with an implantation of a CTR and an IOL (19.5 D; AR40E; Abbott Medical Optics Inc., Santa Ana, CA, USA) in the lens capsule. In March 2011, this patient presented with acute vision decline accompanied by intense coughing and sneezing. Upon ophthalmologic examination, the patient was determined to have a bitemporal-upper quadrant dislocation of the IOL-CTR (Fig. 1). Zonular disruption was observed between the 9 and 2 o'clock positions, suggesting a subluxation of the IOL-CB-CTR complex. At presentation, the patient's vision was 0.2 and intraocular pressure was $15 \mathrm{mmHg}$.

For this patient, repositioning of the IOL by bilaterally suturing the complex to the ciliary groove was proposed. As the patient was placed in a supine position on the operating table, the dislocated IOL-CB-CTR complex migrated to the center of the pupillary axis. The lens dislocation was significantly 
more pronounced when the patient was in a sitting position compared to that observed in a supine position.

Manipulation of the position of the head did not cause any excessive frontal-temporal offset of the IOL-CB-CTR complex. No fractures were present inferior to the nasal zonules and the position of the capsular bag was maintained. The right pupil was dilated by tropicamide, and retrobulbar anesthesia consisting of $2 \mathrm{cc}$ lidocaine and $2 \mathrm{cc}$ bupivacaine was then injected into the retrobulbar space. Subsequently, two 1-mm corneoscleral incisions were made at the 11 and 3 o'clock positions. Sodium hyaluronate $(1 \%)$ was then injected and allowed to move through the vitreous fluid into the posterior chamber. The IOL-CB-CTR complex was moved nasally to expose the tension ring and IOL haptics. A fornix-based conjunctival flap was made. A partial-thickness triangular scleral flap was made 2.0-2.5 $\mathrm{mm}$ posterior to the surgical limbus. A Z-suture was placed on the sclera $4 \mathrm{~mm}$ from the corneoscleral limbus. In this position, one double-curved $10-0$ polypropylene (Prolene) suture was passed through the sclera into the posterior chamber, penetrating the posterior capsules and wrapping perpendicularly around the tangential contact point between the IOL haptics and the CTR, and finally passing through the anterior capsules into the anterior chamber (Fig. 2). The polypropylene suture was passed over and under the CTR, which was then retracted through the sclera. The 10-0 polypropylene suture was pulled to provide adequate tension for the $\mathrm{CB}$ centration.

From the 3 o'clock corneoscleral limbus incision, the stitches were cut from the incision suture and the broken ends were pulled out. The thread was cut near the needle and then pulled out from the incision. The suture lines were pulled out from the anterior and posterior surfaces of the IOL-CB-CTR complex, and a knot was tied $1 \mathrm{~mm}$ away from the corneoscleral incision. The knot was buried in the scleral bed and covered with the scleral flap and conjunctiva. After the sutures were cut, the IOL-CB-CTR complex was repositioned to the center by pulling the suture line (Fig. 3; Video S1).

It is standard knowledge that intact zonules provide support and circular contour to the capsular bag. In the present study, the position of the CTR-CB-IOL complex was maintained through a unilateral suture fixation. No sutures were made on the contralateral side. The last procedure was an anterior vitrectomy, which consisted of the removal of the sac and the structures surrounding the anterior chamber.

One day after the procedure, the patient's vision was 0.4 and the CTR-CB-IOL complex remained centered, with minor bleeding visible in the vitreous space. At two weeks after the procedure, the hematoceles had been reabsorbed. At the one-year follow-up, the patient's vision was 0.8 and the IOL-CB-CTR complex remained centered (Fig. 4).

\section{Discussion}

The present study described a novel method to correct a dislocation and subluxation of an IOL-CTR that avoided explantation of the IOL. Rather, the IOL-CB-CTR complex was simply and effectively immobilized.

Suture immobilization of a subluxated or dislocated IOL is not a novel technique, but it has been underutilized (1). A similar operation was described in one case of CTR-CB-IOL

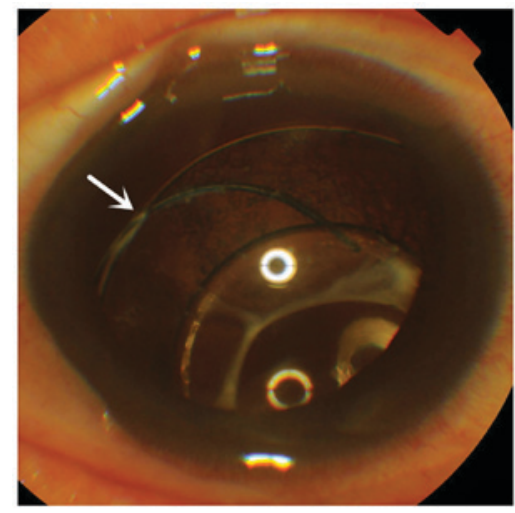

Figure 1. Dislocation of the IOL-capsular bag-CTR complex. One end of the CTR as well as the haptic of the IOL is visible (arrow). CTR, capsular tension ring; IOL, intraocular lens.

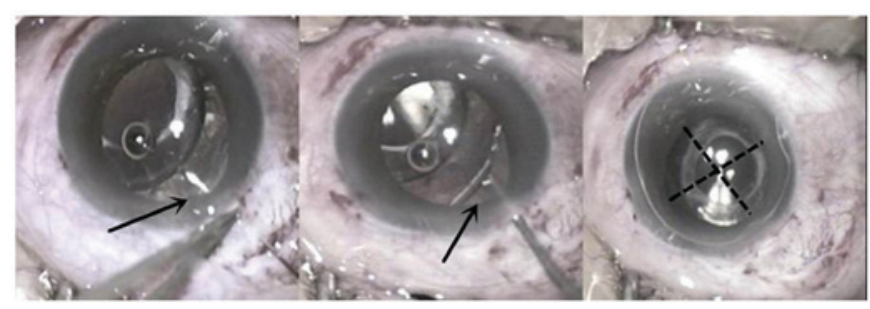

Figure 2. Suturing of the IOL-CB-CTR complex. Left panel: A corneoscleral incision is made and a needle with a 10-0 polypropylene suture (black arrow) is passed through the sclera to reach the IOL-CB-CTR complex. Middle panel: A suture (black arrow) is wrapped around the tangential contact point of the IOL haptic and the CTR. Right panel: The IOL-CB-CTR complex is repositioned to the center of the pupil (black cross). CTR, capsular tension ring; IOL, intraocular lens; CB, capsular bag.

dislocation repair, in which two sutures were placed 180 degrees apart to reposition the IOL-CB-CTR complex (1). By contrast, the present procedure used a single suture through the IOL-CB-CTR complex and the remaining ciliary fibers to provide contralateral support. Oner et al (9) reported a IOL-CB-CTR subluxation suture repair technique involving the transscleral placement of two 10-0 polypropylene sutures over and under the CTR through the anterior and posterior capsular surfaces to secure the CTR. This was then retracted and sutured through the sclera. The present technique involves fewer traumatic needle paths and smaller incisions.

The path of the suture is crucial for determining the degree of scleral penetration, which may be associated with hemorrhage of the ciliary bodies $(10,11)$. Favorable suture paths are only one of the advantages of the present technique. In published reports, at least two punctures were performed in the sclera. However, in our case report, the sclera is penetrated only once. Less amount of penetration avoids any repeated perforations and reduces the risk of hemorrhage. The suture is tied faster than in corresponding techniques $(7,11,12)$. The final fixed position of the IOL-CB-CTR complex is similar to the anatomical position.

As the ideal result, the haptics of the IOL and the tension ring would be fixed precisely within the ciliary groove. The Z-suture between the sclera has sufficient tension to fix the complex, and the inflammatory adhesion produced by the suture and sclera supports this. Using the present method 


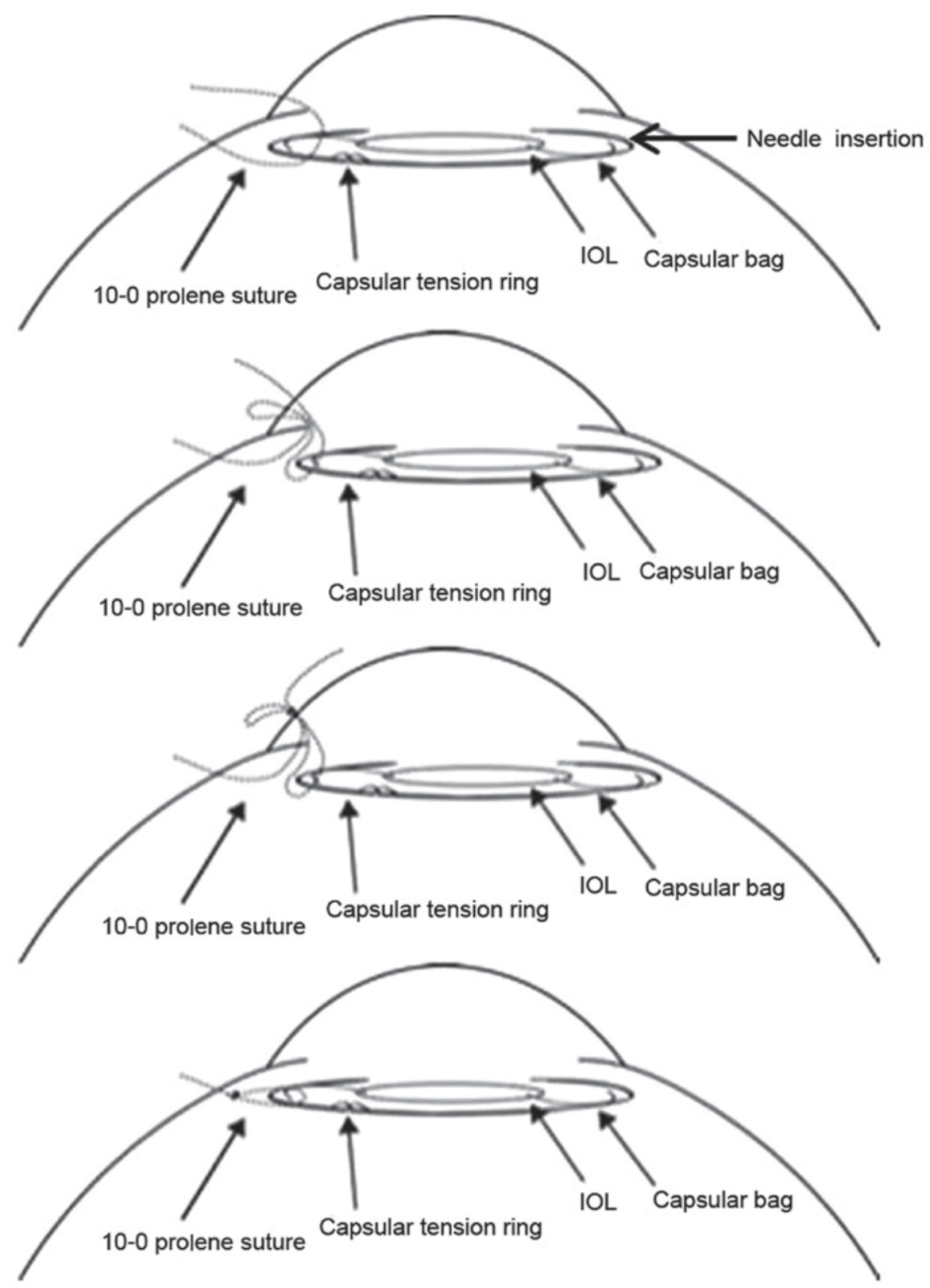

Figure 3. A 10-0 polypropylene suture is hooked perpendicularly around the tangential point of the IOL haptic and the capsular tension ring. Through a 1-mm corneoscleral incision, a $\mathrm{Z}$ suture is formed and tied, leaving no sutures penetrating the corneoscleral limbus. IOL, intraocular lens.

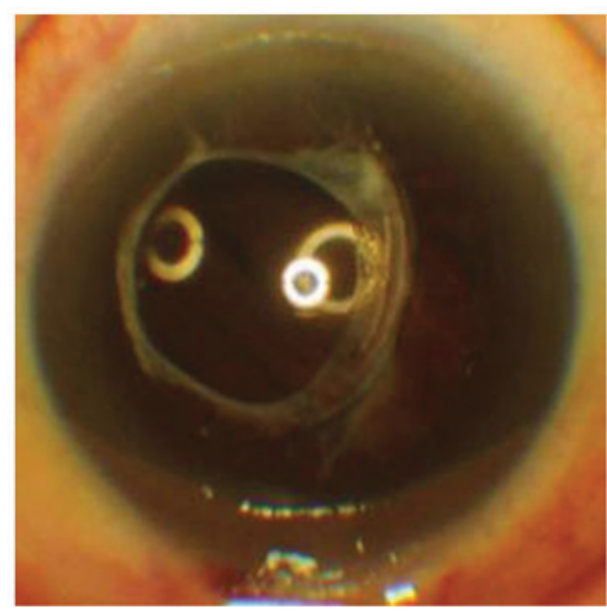

Figure 4. At the one-year follow-up, the capsular tension ring and intraocular lens remain in the middle of the pupil and no further dislocation had occurred. the creation of partial-thickness triangular scleral flaps with low intraocular pressure is not required, thereby reducing the time of the procedure, which may in turn reduce the rate of complications and endophthalmitis. This method may prevent future dislocations of the IOL-CB-CTR complex, as the tension of the suture may be adjusted even after the suture has been knotted. In this way, the present technique simplifies the repositioning of the IOL-CB-CTR complex. Another advantage of this technique is the minimally invasive $1-\mathrm{mm}$ corneoscleral limbus incision, which reduces the risk of astigmatism. The small incision keeps the anterior chamber stable and prevents partial or complete movement during suturing of the complex.

Regardless of the type of suture, the position of the suture that penetrates the capsule and its relative orientation with the IOL-CTR contact point determine the success of the procedure. The tension ring and IOL haptics are independent 
circular structures and physically meet nearly tangentially inside the capsular bag. The suture should penetrate the capsule through a point perpendicular to the tangential IOL-CTR contact point. Based on the authors' experience, if the suture is inserted away from this point, the capsule may be torn by the suture, bearing a risk of spillage of the IOL into the vitreous cavity. If the suture only secures the CTR, it cannot adequately support the stability of the entire complex, as the tension of the suture is unbalanced. Of note, pre-operative evaluation of the zonules should be performed to determine whether unilateral or bilateral suturing is required.

In conclusion, suturing the CTR-CB-IOL complex is simple and effective. It secures the position of the IOL and prevents any requirement for the explantation of the complex or suturing of a new IOL onto the sclera, increasing the work efficiency of the surgery.

\section{Acknowledgements}

Not applicable.

\section{Funding}

No funding received.

\section{Availability of data and materials}

The data and materials in this study are available from the corresponding author on reasonable request.

\section{Authors' contributions}

LG collected, collated and classified the experimental data, and wrote the manuscript. XY responsible for the application of experimental ethics. CW performed the surgeries. All three authors designed the current study, and analysed and interpreted the data.

\section{Ethical approval and consent to participate}

The present study was approved by the Ethics Committee of Xi'an No. 4 Hospital, Affiliated GuangRen Hospital, School of Medicine, Xi'an Jiao Tong University (Xi'an, China).

\section{Patient consent for publication}

The patient provided informed consent for the publication of the intra-operative images/video.

\section{Competing interests}

None of the authors has any conflicts of interest to declare.

\section{References}

1. Moreno-Montañés J, Heras H and Fernández-Hortelano A: Surgical treatment of a dislocated intraocular lens-capsular bag-capsular tension ring complex. J Cataract Refract Surg 31: 270-273, 2005.

2. Bhattacharjee H, Bhattacharjee K, Das D, Jain PK, Chakraborty D and Deka S: Management of a posteriorly dislocated endocapsular tension ring and a foldable acrylic intraocular lens. J Cataract Refract Surg 30: 243-246, 2004.

3. Gross JG, Kokame GT and Weinberg DV; Dislocated In-The-Bag Intraocular Lens Study Group: In-the-bag intraocular lens dislocation. Am J Ophthalmol 137: 630-635, 2004.

4. Scherer M, Bertelmann E and Rieck P: Late spontaneous in-the-bag intraocular lens and capsular tension ring dislocation in pseudoexfoliation syndrome. J Cataract Refract Surg 32: 672-675, 2006.

5. Ahmed II,Chen SH, Kranemann C and Wong DT: Surgical repositioning of dislocated capsular tension rings. Ophthalmology 112: 1725-1733, 2005.

6. Werner L, Zaugg B, Neuhann T, Burrow M and Tetz M: In-the-bag capsular tension ring and intraocular lens subluxation or dislocation: A series of 23 cases. Ophthalmology 119: 266-271, 2012.

7. Tribus C, Alge CS, Haritoglou C, Lackerbauer C, Kampik A, Mueller A and Priglinger SG: Indications and clinical outcome of capsular tension ring (CTR) implantation: A review of 9528 cataract surgeries. Clin Ophthalmol 1: 65-69, 2007.

8. Lang Y, Fineberg E and Garzozi HJ: Vitrectomy to remove a posteriorly dislocated endocapsular tension ring. J Cataract Refract Surg 27: 474-476, 2001.

9. Oner FH, Kocak N and Saatci AO: Dislocation of capsular bag with intraocular lens and capsular tension ring. J Cataract Refract Surg 32: 1756-1758, 2006.

10. Yang CS and Chao YJ: Long-term outcome of combined vitrectomy and transscleral suture fixation of posterior chamber intraocular lenses in the management of posteriorly dislocated lenses. J Chin Med Assoc 79: 450-455, 2016.

11. Banaee T and Sagheb S: Scleral fixation of intraocular lens in eyes with history of open globe injury. J Pediatr Ophthalmol Strabismus 48: 292-297, 2011.

12. John T, Tighe S, Hashem O and Sheha H: New use of 8-0 polypropylene suture for four-point scleral fixation of secondary intraocular lenses. J Cataract Refract Surg 44: 1421-1425, 2018. 